\title{
Construction of Frames on the Heisenberg Groups
}

https://doi.org/10.1515/agms-2020-0118

Received August 7, 2020; accepted November 23, 2020

Abstract: In this paper, we present a construction of frames on the Heisenberg group without using the Fourier transform. Our methods are based on the Calderón-Zygmund operator theory and Coifman's decomposition of the identity operator on the Heisenberg group. These methods are expected to be used in further studies of several complex variables.

Keywords: Heisenberg group; frame; Calderón-Zygmund singular integral operator; Coifman's decomposition of the identity operator

MSC: 42B20, 42B30

\section{Introduction}

The theory of wavelet analysis has played an important role in many different branches of science and technology; see, for instance, $[1,2,7,8,11]$ and the references therein. Wavelet analysis provides a simpler and more efficient way to analyze functions and distributions that have been studied through Fourier series and integrals. R. Coifman and G. Weiss invented the atoms and molecules (cf. [6, 21]) which formed the basic building blocks of various function spaces. The atom decomposition can be obtained by using a discrete version of a well-known identity, due to A. Calderón ([3]), in which wavelets were implicitly involved. The wavelet series decompositions are nowadays effective expansion by unconditional bases in various function spaces arising from the theory of harmonic analysis.

Let us now recall the frame constructed by Frazier and Jawerth in [12] using the Fourier transform. Let $\psi \in \mathcal{S}\left(\mathbb{R}^{n}\right)$ with

(i) $\operatorname{supp} \widehat{\varphi} \subset\{1 / 2 \leq|\xi| \leq 2\}$,

(ii) $\sum_{j \in \mathbb{Z}}\left|\widehat{\varphi}\left(2^{-j} \xi\right)\right|^{2}=1, \quad$ for all $\xi \neq 0$.

Then

$$
f(x)=\sum_{j \in \mathbb{Z}} \sum_{Q: \ell(Q)=2^{-j}} 2^{-j n} \varphi_{j}\left(x-x_{Q}\right) \varphi_{j}^{\prime}{ }^{\star} f\left(x_{Q}\right), \quad \forall f \in L^{2}\left(\mathbb{R}^{n}\right),
$$

where $Q$ represents a dyadic cube with $\ell(Q)=2^{-j}, x_{Q}$ represents the lower-left corner of $Q, \varphi_{j}(x)=2^{j n} \varphi\left(2^{j} x\right)$, $\varphi_{j}^{\prime}(x)=2^{j n} \overline{\varphi\left(-2^{j} x\right)}$, and the series converges in $L^{2}\left(\mathbb{R}^{n}\right)$.

Note that the Frazier-Jawarth approach relies on the Fourier transform on Euclidean spaces. However, there are many non-Euclidean situations (for instance, the Ahlfors-type spaces and Carnot-Carathédory-type spaces) in which the Fourier transform is not available. In [4], we presented a new approach for constructing a

Der-Chen Chang: Department of Mathematics and Statistics, Georgetown University, Washington DC, USA; and Graduate Institute of Business Administration, Fu Jen Catholic University, New Taipei City, Taiwan, E-mail: chang@georgetown.edu Yongsheng Han: Department of Mathematics and Statistics, Auburn University, Auburn, USA, E-mail: hanyong@auburn.edu *Corresponding Author: Xinfeng Wu: Department of Mathematics, China University of Mining \& Technology, Beijing, China, E-mail: wuxf@cumtb.edu.cn 
frame without using the Fourier transform on $\mathbb{R}^{n}$. The purpose of this article is to adapt that idea to construct a frame on the Heisenberg group $\mathbb{H}^{n}$.

Let us first recall the classical Calderón reproducing formula established by Geller and Mayeli [13] on the Heisenberg group via the Fourier transform.

Theorem A. (Corollary 1 of [13]) There is $\psi \in C^{\infty}\left(\mathbb{H}^{n}\right)$ satisfying

$$
\begin{aligned}
\text { either } \psi & \in \mathcal{S}\left(\mathbb{H}^{n}\right) \text { and all moments of } \psi \text { vanish, } \\
\text { or } \psi & \in C_{c}^{\infty}\left(\mathbb{H}^{n}\right) \text { and all arbitrarily large moments of } \psi \text { vanish, }
\end{aligned}
$$

such that the following Calderón reproducing formula holds:

$$
f=\int_{0}^{\infty} \psi_{s}^{\vee} \psi_{s}^{\star} f \frac{d s}{s}, \quad f \in L^{2}\left(\mathbb{H}^{n}\right),
$$

where * is the Heisenberg convolution, $\psi^{\vee}(\zeta)=\overline{\psi\left(\zeta^{-1}\right)}$, and $\psi_{s}(z, t)=s^{-2 n-2} \psi\left(\frac{z}{s}, \frac{u}{s^{2}}\right)$ for $s>0$.

Based on this Calderón reproducing formula, Han-Lu-Sawyer [16] established a discrete and wavelet-like Calderón reproducing formula.

Here we use another approach, beginning with an approximation to the identity.

Definition 1.1 (Test function space). A function $f$ defined on $\mathbb{H}^{n}$ is said to be a test function if there exist $\beta, \gamma$, and $r$ with $0<\beta \leq 1$, and $\gamma, r>0$ and $\left(z_{0}, t_{0}\right) \in \mathbb{H}^{n}$ such that

(i) $|f(z, t)| \leq C \frac{r^{\gamma}}{\left(r+d\left((z, t),\left(z_{0}, t_{0}\right)\right)\right)^{2 n+2+\gamma}}$,

(ii) $\left|f(z, t)-f\left(z^{\prime}, t^{\prime}\right)\right| \leq C\left(\frac{d\left((z, t),\left(z^{\prime}, t^{\prime}\right)\right)}{r+d\left((z, t),\left(z_{0}, t_{0}\right)\right)}\right)^{\beta} \frac{r^{\gamma}}{\left(r+d\left((z, t),\left(z_{0}, t_{0}\right)\right)\right)^{2 n+2+\gamma}}$ for $d\left((z, t),\left(z^{\prime}, t^{\prime}\right)\right) \leq(1 / 2 a)(r+$ $\left.d\left((z, t),\left(z_{0}, t_{0}\right)\right)\right)$

(iii) $\int_{\mathbb{H}^{n}} f(z, t) d z d t=0$,

where $a$ is the constant in the quasi-triangle inequality (see Section 2 below).

If $f$ is a test function as above, we denote $f \in \mathcal{M}\left(\beta, \gamma, r, z_{0}, t_{0}\right)$ and the norm of $f \in \mathcal{M}\left(\beta, \gamma, r, z_{0}, t_{0}\right)$ is defined to be the smallest constant $C$ given in (i) and (ii) above.

Denote $\mathcal{M}(\beta, \gamma, r)=\mathcal{M}(\beta, \gamma, r, 0,0)$ and $\mathcal{M}(\beta, \gamma)=\mathcal{M}(\beta, \gamma, 1)$. It is easy to see that $\mathcal{M}\left(\beta, \gamma, r, z_{0}, t_{0}\right)=$ $\mathcal{M}(\beta, \gamma)$ with equivalent norms for all $\left(z_{0}, t_{0}\right) \in \mathbb{H}^{n}$ and $r>0$. Furthermore, it is also easy to check that $\mathcal{M}(\beta, \gamma)$ is a Banach space with respect to the norm in $\mathcal{N}(\beta, \gamma)$. We refer the reader to $[11,17,18]$ for more details.

The following is our main result, whose proof is based on the Calderón-Zygmund operator theory.

Theorem 1.2. Suppose that $\phi(z, t)$ is a function satisfying the above conditions (i) and (ii) with $\int_{\mathbb{H}^{n}} \phi(z, t) d z d t=1$. Set $\psi(z, t)=\phi(z, t)-\phi_{1}(z, t)$ where $f_{j}(z, t)=2^{j(2 n+2)} f\left(2^{j} z, 2^{2 j} t\right)$. Then there exist families of functions $\widetilde{\psi}_{j}(z, t ; w, s)$ and $\widetilde{\psi}_{j}^{*}(z, t ; w, s)$ such that for all $f \in L^{2}\left(\mathbb{H}^{n}\right)$,

$$
\begin{aligned}
f(z, t) & =\sum_{j \in \mathbb{Z}} \sum_{\substack{Q-j \\
\ell(Q)=2^{-j-N}}}|Q| \widetilde{\psi}_{j}\left(z, t, z_{Q}, t_{Q}\right) \psi_{j}{ }^{\star} f\left(z_{Q}, t_{Q}\right) \\
& =\sum_{j \in \mathbb{Z}} \sum_{\substack{Q \\
\ell(Q)=2^{-j-N}}}|Q| \psi_{j}\left((z, t) \cdot\left(z_{Q}, t_{Q}\right)^{-1}\right) \widetilde{\psi}_{j}^{\star}(f)\left(z_{Q}, t_{Q}\right),
\end{aligned}
$$

where $Q$ represents a dyadic cube on $\mathbb{H}^{n}$ of side length $\ell(Q)=2^{-j-N}$ with $N$ being a large fixed positive integer, $\left(z_{Q}, t_{Q}\right)$ is any fixed point in $Q$,

$$
\widetilde{\psi}_{j}^{\star}(f)\left(z_{Q}, t_{Q}\right)=\int_{\mathbb{H}^{n}} \widetilde{\psi}_{j}^{\star}\left(z_{Q}, t_{Q}, w, s\right) f(w, s) d w d s,
$$


and $\widetilde{\psi}_{j}\left(z, t, z_{Q}, t_{Q}\right)$ and $\widetilde{\psi}_{j}^{\star}\left(z_{Q}, t_{Q}, z, t\right)$ satisfy the similar smoothness and cancellation conditions as $\psi_{j}((z, t)$. $\left.\left(z_{Q}, t_{Q}\right)^{-1}\right)$.

For clarification purposes, we point out that the number $N$ must be sufficiently large, depending on $\phi$ and the dimension. As mentioned before, the key tool used in the proof for Frazier-Jawerth's result is the Fourier transform. In contrast, Theorem 1.2 is proven through the Calderón-Zygmund operator theory.

Throughout the article, let $X \lesssim Y$ mean that there is a constant $c>0$ such that $X \leq c Y$.

This article is organized as follows. In Section 2, we present preliminaries and some lemmas. The main result, Theorem 1.2 is proven in Section 3.

\section{Preliminaries and some lemmas}

The $n$-dimensional Heisenberg group $\mathbb{H}^{n}$ consists of the set $\mathbb{C}^{n} \times \mathbb{R}=\left\{(z, t): z \in \mathbb{C}^{n}, t \in \mathbb{R}\right\}$ with the multiplication law

$$
(z, t) \cdot(w, s)=(z+w, t+s+2 \operatorname{Im}(z \bar{w})) .
$$

The Haar measure on $\mathbb{H}^{n}$ is just the Lebesgue measure $d z d t$. The convolution on $\mathbb{H}^{n}$ is defined by

$$
\begin{aligned}
f \star g(z, t) & =\int_{\mathbb{H}^{n}} f(w, s) g\left((w, s)^{-1} \cdot(z, t)\right) d w d s \\
& =\int_{\mathbb{H}^{n}} f\left((z, t) \cdot(w, s)^{-1}\right) g(w, s) d w d s .
\end{aligned}
$$

The nonisotropic dilation on $\mathbb{H}^{n}$ is defined by $\delta \circ(z, t)=\left(\delta z, \delta^{2} t\right), \forall \delta>0$. These dilations are automorphisms of $\mathbb{H}^{n}$ :

$$
\delta \circ((z, t) \cdot(w, s))=(\delta \circ(z, t)) \cdot(\delta \circ(w, s)) .
$$

A homogeneous norm on $\mathbb{H}^{n}$ is given by $\rho((z, t))=\left(|z|^{4}+t^{2}\right)^{\frac{1}{4}}$. The quasi-distance $d$ on $\mathbb{H}^{n}$, induced by $\rho$, is defined by

$$
d((z, t),(w, s))=\rho\left((w, s)^{-1} \cdot(z, t)\right),
$$

which satisfies the quasi-triangle inequality

$$
d\left((z, t),\left(z^{\prime}, t^{\prime}\right)\right) \leq a\left[d((z, t),(w, s))+d\left((w, s),\left(z^{\prime}, t^{\prime}\right)\right)\right]
$$

We define balls $B((z, t), \delta)$ in $\mathbb{H}^{n}$ by

$$
B((z, t), \delta)=\left\{(w, s) \in \mathbb{H}^{n}: d((w, s),(z, t))<\delta\right\}
$$

These balls are left-invariant under the action of $\mathbb{H}^{n}$. We refer the reader to Stein [20] for more background about the Heisenberg groups. Notice that $\left(\mathbb{H}^{n}, \rho, d z d t\right)$ is a space of homogeneous type in the sense of Coifman and Weiss [9].

Christ [5] provided a dyadic grid in a space of homogeneous type.

Lemma 2.1. Let $X$ be a space of homogeneous type. Then there exists a collection $\left\{I_{\alpha}^{k} \subset X: k \in \mathbb{Z}, \alpha \in J^{k}\right\}$ of open subsets, where $\mathrm{J}^{k}$ is some index set, and $C_{1}, C_{2}>0$, such that

(i) $\mu\left(X \backslash \cup_{\alpha} I_{\alpha}^{k}\right)=0$ for each fixed $k$ and $I_{\alpha}^{k} \cap I_{\beta}^{k}=\emptyset$, if $\alpha=\beta$;

(ii) for any $\alpha, \beta, k, l$ with $l \geq k$, either $I_{\beta}^{l} \subset I_{\alpha}^{k}$ or $I_{\beta}^{l} \cap I_{\alpha}^{k}=\emptyset$;

(iii) for each $(k, \alpha)$ and each $l \leq k$, there is a unique $\beta$ such that $I_{\alpha}^{k} \subset I_{\beta}^{l}$;

(iv) $\operatorname{diam}\left(I_{\alpha}^{k}\right) \leq C_{1} 2^{-k}$;

(v) each $I_{\alpha}^{k}$ contains some ball $B\left(z_{\alpha}^{k}, C_{2} 2^{-k}\right)$, where $z_{\alpha}^{k} \in X$. 
We may think of $I_{\alpha}^{k}$ as being a dyadic cube with side-length $\ell\left(I_{\alpha}^{k}\right)=2^{-k}$ centered at $z_{\alpha}^{k}$.

The following is the definition of generalized approximations to the identity of the Heisenberg group, whose kernels have only Lipschitz smoothness.

Definition 2.2. Let $0<\epsilon \leq 1$. A sequence $\left\{S_{k}\right\}_{k \in \mathbb{Z}}$ of operators is said to be an $\epsilon$-approximation to the identity (or simply an approximation to the identity) if there exists $0<C<\infty$ such that for all $(z, t),(w, s) \in$ $\mathbb{H}^{n}, S_{k}(z, t ; w, s)$, the kernel of $S_{k}$, are functions from $\mathbb{H}^{n} \times \mathbb{H}^{n}$ into $\mathbb{C}$ satisfying

$$
\begin{gathered}
\left|S_{k}(z, t ; w, s)\right| \leq C \frac{2^{-k \epsilon}}{\left(2^{-k}+d((z, t),(w, s))\right)^{2 n+2+\epsilon}} ; \\
\left|S_{k}(z, t ; w, s)-S_{k}\left(z^{\prime}, t^{\prime} ; w, s\right)\right| \lesssim\left(\frac{d\left((z, t),\left(z^{\prime}, t^{\prime}\right)\right)}{2^{-k}+d((z, t),(w, s))}\right)^{\epsilon} \frac{2^{-k \epsilon}}{\left(2^{-k}+d((z, t),(w, s))\right)^{2 n+2+\epsilon}}
\end{gathered}
$$

for $d\left((z, t),\left(z^{\prime}, t^{\prime}\right)\right) \leq \frac{1}{2 a}\left(2^{-k}+d((z, t),(w, s))\right)$;

$$
\left|S_{k}(z, t ; w, s)-S_{k}\left(z, t ; w^{\prime}, s^{\prime}\right)\right| \leq C\left(\frac{d\left((w, s),\left(w^{\prime}, s^{\prime}\right)\right)}{2^{-k}+d((z, t),(w, s))}\right)^{\epsilon} \frac{2^{-k \epsilon}}{\left(2^{-k}+d((z, t),(w, s))\right)^{2 n+2+\epsilon}}
$$

for $d\left((w, s),\left(w^{\prime}, s^{\prime}\right)\right) \leq \frac{1}{2 a}\left(2^{-k}+d((z, t),(w, s))\right)$;

$$
\int_{\mathbb{H}^{n}} S_{k}(z, t ; w, s) d w d s=1
$$

for all $(z, t) \in \mathbb{H}^{n}$;

$$
\int_{\mathbb{H}^{n}} S_{k}(z, t ; w, s) d z d t=1
$$

for all $(w, s) \in \mathbb{H}^{n}$.

Note that if $\phi(z, t)$ is a function satisfying the above conditions (i) and (ii) with $\int_{\mathbb{H}^{n}} \phi(z, t) d z d t=1$, then $\phi_{k}\left((z, t) \cdot(w, s)^{-1}\right)$ is an $\epsilon$-approximation to the identity. Let $D_{k}=S_{k}-S_{k-1}$ and $D_{k}(x, y)$ be the kernel for $k \in \mathbb{Z}$. Then $D_{k}(\cdot, y) \in \mathcal{M}\left(\epsilon, \epsilon, 2^{-k}\right)$ for any fixed $y=(w, s)$ and $k$, and similarly, $D_{k}(x, \cdot) \in \mathcal{M}\left(\epsilon, \epsilon, 2^{-k}\right)$ for any fixed $x=(z, t)$ and $k$.

The following result follows from a more general one in [11, Lemmas 3.7 and 3.11].

Lemma 2.3. Let $0<\epsilon \leq 1$. Suppose that $\left\{\phi_{k}\right\}_{k \in \mathbb{Z}}$ is an $\epsilon$-approximation to the identity. Set $\psi_{k}=\phi_{k}-\phi_{k-1}$ for all $k \in \mathbb{Z}$. Then for $0<\epsilon^{\prime}<\epsilon$, there exists a constant $C$ which depends on $\epsilon^{\prime}$ and $\epsilon$, but not on $k$, l such that

$$
\left|\psi_{k}{ }^{\star} \psi_{l}\left((z, t) \cdot(w, s)^{-1}\right)\right| \leq C 2^{-|k-l| \epsilon^{\prime}} \frac{2^{-(k \wedge l) \epsilon}}{\left(2^{-(k \wedge l)}+d((z, t),(w, s))\right)^{2 n+2+\epsilon}} ;
$$

for $k \geq l$ and $d\left((z, t),\left(z^{\prime}, t^{\prime}\right)\right) \leq \frac{1}{2 a}\left(2^{-l}+d((z, t),(w, s))\right)$

$$
\left|\psi_{k} \star \psi_{l}\left((z, t) \cdot(w, s)^{-1}\right)-\psi_{k} \star \psi_{l}\left(\left(z^{\prime}, t^{\prime}\right) \cdot(w, s)^{-1}\right)\right| \leq C\left(\frac{d\left((z, t),\left(z^{\prime}, t^{\prime}\right)\right)}{2^{-l}+d((z, t),(w, s))}\right)^{\epsilon} \frac{2^{-l \epsilon}}{\left(2^{-l}+d((z, t),(w, s))\right)^{2 n+2+\epsilon}} ;
$$

for $k \leq l$ and $d\left((z, t),\left(z^{\prime}, t^{\prime}\right)\right) \leq \frac{1}{2 a}\left(2^{-k}+d((z, t),(w, s))\right)$

$\left|\psi_{k}{ }^{\star} \psi_{l}\left((z, t) \cdot(w, s)^{-1}\right)-\psi_{k}{ }^{\star} \psi_{l}\left(\left(z^{\prime}, t^{\prime}\right) \cdot(w, s)^{-1}\right)\right| \leq C\left(\frac{d\left((z, t),\left(z^{\prime}, t^{\prime}\right)\right)}{2^{-k}+d((z, t),(w, s))}\right)^{\epsilon^{\prime}} \frac{2^{-k\left(\epsilon-\epsilon^{\prime}\right)}}{\left(2^{-k}+d((z, t),(w, s))\right)^{2 n+2+\epsilon-\epsilon^{\prime}}} ;$

for $k \leq l$ and $d\left((w, s),\left(w^{\prime}, s^{\prime}\right)\right) \leq \frac{1}{2 a}\left(2^{-k}+d((z, t),(w, s))\right)$

$\left|\psi_{k}{ }^{\star} \psi_{l}\left((z, t) \cdot(w, s)^{-1}\right)-\psi_{k}{ }^{\star} \psi_{l}\left((z, t) \cdot\left(w^{\prime}, s^{\prime}\right)^{-1}\right)\right| \leq C\left(\frac{d\left((w, s),\left(w^{\prime}, s^{\prime}\right)\right)}{2^{-k}+d((z, t),(w, s))}\right)^{\epsilon} \frac{2^{-k \epsilon}}{\left(2^{-k}+d((z, t),(w, s))\right)^{2 n+2+\epsilon}} ;$

for $l \leq k$ and $d\left((w, s),\left(w^{\prime}, s^{\prime}\right)\right) \leq \frac{1}{2 a}\left(2^{-l}+d((z, t),(w, s))\right)$

$\left|\psi_{k}{ }^{\star} \psi_{l}\left((z, t) \cdot(w, s)^{-1}\right)-\psi_{k}{ }^{\star} \psi_{l}\left((z, t) \cdot\left(w^{\prime}, s^{\prime}\right)^{-1}\right)\right| \leq C\left(\frac{d\left((w, s),\left(w^{\prime}, s^{\prime}\right)\right)}{2^{-l}+d((z, t),(w, s))}\right)^{\epsilon^{\prime}} \frac{2^{-l\left(\epsilon-\epsilon^{\prime}\right)}}{\left(2^{-l}+d((z, t),(w, s))\right)^{2 n+2+\epsilon-\epsilon^{\prime}}}$. 
Definition 2.4. An approximation to the identity $\left\{S_{k}\right\}_{k \in \mathbb{Z}}$ is said to satisfy the double Lipschitz condition if

$$
\begin{aligned}
& \left|S_{k}(z, t ; w, s)-S_{k}\left(z^{\prime}, t^{\prime} ; w, s\right)-S_{k}\left(z, t ; w^{\prime}, s^{\prime}\right)+S_{k}\left(z^{\prime}, t^{\prime} ; w^{\prime}, s^{\prime}\right)\right| \\
& \quad \lesssim\left(\frac{d\left((z, t),\left(z^{\prime}, t^{\prime}\right)\right)}{2^{-k}+d((z, t),(w, s))}\right)^{\epsilon}\left(\frac{d\left((w, s),\left(w^{\prime}, s^{\prime}\right)\right)}{2^{-k}+d((z, t),(w, s))}\right)^{\epsilon} \frac{2^{-k \epsilon}}{\left(2^{-k}+d((z, t),(w, s))\right)^{2 n+2+\epsilon}}
\end{aligned}
$$

for $d\left((z, t),\left(z^{\prime}, t^{\prime}\right)\right) \leq \frac{1}{2 a}\left(2^{-k}+d((z, t),(w, s))\right)$ and $d\left((w, s),\left(w^{\prime}, s^{\prime}\right)\right) \leq \frac{1}{2 a}\left(2^{-k}+d((z, t),(w, s))\right)$.

Lemma 2.5. [11, Lemma 3.12] Suppose that $\left\{S_{k}\right\}_{k \in \mathbb{Z}}$ is an approximation to the identity and $S_{k}((z, t),(w, s))$, the kernels of $S_{k}$, satisfy the condition (2.6). Set $D_{k}=S_{k}-S_{k-1}$ for all $k \in \mathbb{Z}$. Then for any $0<\epsilon^{\prime}<\epsilon$, there exists a constant $C$ which depends on $\epsilon^{\prime}$ and $\epsilon$, but not on $k$ or $l$, such that

$$
\begin{aligned}
\left|\psi_{k}{ }^{\star} \psi_{l}\left((z, t) \cdot(w, s)^{-1}\right)-\psi_{k}{ }^{\star} \psi_{l}\left(\left(z^{\prime}, t^{\prime}\right) \cdot(w, s)^{-1}\right)-\psi_{k}{ }^{\star} \psi_{l}\left((z, t) \cdot\left(w^{\prime}, s^{\prime}\right)^{-1}\right)+\psi_{k}{ }^{*} \psi_{l}\left(\left(z^{\prime}, t^{\prime}\right) \cdot\left(w^{\prime}, s^{\prime}\right)^{-1}\right)\right| \\
\leq C\left(\frac{d\left((z, t),\left(z^{\prime}, t^{\prime}\right)\right)}{2^{-(k \wedge l)}+d((z, t),(w, s))}\right)^{\epsilon^{\prime}}\left(\frac{d\left((w, s),\left(w^{\prime}, s^{\prime}\right)\right)}{2^{-(k \wedge l)}+d((z, t),(w, s))}\right)^{\epsilon^{\prime}} \frac{2^{-(k \wedge l)\left(\epsilon-\epsilon^{\prime}\right)}}{\left(2^{-(k \wedge l)}+d((z, t),(w, s))\right)^{2 n+2+\epsilon-\epsilon^{\prime}}}
\end{aligned}
$$

for $d\left((z, t),\left(z^{\prime}, t^{\prime}\right)\right) \leq \frac{1}{2 a}\left(2^{-(k \wedge l)}+d((z, t),(w, s))\right)$ and $d\left((w, s),\left(w^{\prime}, s^{\prime}\right)\right) \leq \frac{1}{2 a}\left(2^{-(k \wedge l)}+d((z, t),(w, s))\right)$.

Definition 2.6. Let $\mathcal{D}\left(\mathbb{R}^{n}\right)$ be the space of smooth functions on $\mathbb{H}^{n}$ with compact support, and let $\mathcal{D}^{\prime}\left(\mathbb{H}^{n}\right)$ denote its dual space. An operator $T$ is said to be a Calderón-Zygmund singular integral operator with the kernel $K$ if $T: \mathcal{D}\left(\mathbb{H}^{n}\right) \rightarrow \mathcal{D}^{\prime}\left(\mathbb{H}^{n}\right)$ with

$$
\langle T f, g\rangle=\int_{\mathbb{H}^{n}} \int_{\mathbb{H}^{n}} K(z, t ; w, s) f(w, s) g(z, t) d z d t d w d s,
$$

for $f, g \in \mathcal{D}\left(\mathbb{H}^{n}\right)$ with supp $f \cap$ supp $g=\varnothing$, where the kernel $K(z, t ; w, s)$ is a complex-valued continuous function on $\mathbb{H}^{n} \times \mathbb{H}^{n} \backslash\{(z, t)=(w, s)\}$ and satisfies the following estimates: For some $0<\epsilon \leq 1$ and some $c>1$,

(i) $|K(z, t ; w, s)| \leq \frac{C}{d((z, t),(w, s))^{2 n+2}}$ for $(z, t) \neq(w, s)$,

(ii) $\left|K(z, t ; w, s)-K\left(z^{\prime}, t^{\prime} ; w, s\right)\right| \leq C \frac{d\left((z, t),\left(z^{\prime}, t^{\prime}\right)\right)^{\epsilon}}{d((z, t),(w, s))^{2 n+2+\epsilon}}$ for $d\left((z, t),\left(z^{\prime}, t^{\prime}\right)\right) \leq(1 / 2 c) d((z, t),(w, s))$,

(iii) $\left|K(z, t ; w, s)-K\left(z, t ; w^{\prime}, s^{\prime}\right)\right| \leq C \frac{d\left((w, s),\left(w^{\prime}, s^{\prime}\right)\right)^{\epsilon}}{d((z, t),(w, s))^{2 n+2+e}}$ for $d\left((w, s),\left(w^{\prime}, s^{\prime}\right)\right) \leq(1 / 2 c) d((z, t),(w, s))$.

The main tool we will use to show Theorem 1.2 is the following result.

Theorem 2.7. [15] Suppose that $T$ is a Calderón-Zygmund singular integral operator and extends to be a bounded operator on $L^{2}\left(\mathbb{H}^{n}\right)$. Furthermore, if $T(1)=T^{\star}(1)=0$ and the kernel $K(z, t ; w, s)$ satisfies the following double difference condition:

$$
\left|\left[K(z, t ; w, s)-K\left(z^{\prime}, t^{\prime} ; w, s\right)\right]-\left[K\left(z, t ; w^{\prime}, s^{\prime}\right)-K\left(z^{\prime}, t^{\prime} ; w^{\prime}, s^{\prime}\right)\right]\right| \lesssim \frac{d\left((z, t),\left(z^{\prime}, t^{\prime}\right)\right)^{\epsilon} d\left((w, s),\left(w^{\prime}, s^{\prime}\right)\right)^{\epsilon}}{d((z, t),(w, s))^{2 n+2+2 \epsilon}}
$$

for $d\left((z, t),\left(z^{\prime}, t^{\prime}\right)\right) \leq \frac{1}{4 a} d((z, t),(w, s))$ and $d\left((w, s),\left(w^{\prime}, s^{\prime}\right)\right) \leq \frac{1}{4 a} d((z, t),(w, s))$, then $T$ maps $\mathcal{M}(\beta, \gamma, r)$ to $\mathcal{M}(\beta, \gamma, r)$ for $0<\beta, \gamma<\epsilon$, and all $\gamma>0$. Moreover, there exists a constant $C$ such that

$$
\|T f\|_{\mathcal{M}(\beta, \gamma, r)} \leq C\|T\| \cdot\|f\|_{\mathcal{M}(\beta, \gamma, r)},
$$

where $\|T\|=\|T\|_{L^{2} \rightarrow L^{2}}+\|T\|_{C Z}$, the $\|T\|_{C Z}$ being the smallest constants in the definition of Calderón-Zygmund kernels and (2.7).

We would also like to mention a result of Meyer. To study the property of the Besov space $\dot{B}_{1}^{0,1}\left(\mathbb{R}^{n}\right)$, Meyer introduced the following definition of smooth atoms.

Definition 2.8. [19] A function $f(x)$ is said to be a smooth atom if there exist $0<\beta \leq 1, \gamma, r>0$, and a constant C such that 
(i) $|f(x)| \leq C \frac{r^{\gamma}}{(r+|x|)^{n+\gamma}}$,

(ii) $\left|f(x)-f\left(x^{\prime}\right)\right| \leq C\left(\frac{\left|x-x^{\prime}\right|}{r}\right)^{\beta}\left[\frac{r^{\gamma}}{(r+|x|)^{n+\gamma}}+\frac{r^{\gamma}}{\left(r+\mid x^{\prime}\right)^{n+\gamma}}\right]$,

(iii) $\int_{\mathbb{R}^{n}} f(x) d x=0$.

If $f$ is a smooth atom as above, then the norm of $f$ is defined by the smallest constant $C$ given in (i) and (ii) above and is denoted by $\|f\|_{M(\beta, \gamma, r)}$.

We would like to point out that if $f \in \mathcal{M}(\beta, \gamma, r)$, then $f \in M(\beta, \gamma, r)$. Meyer in [19] proved the following theorem. The extension of this result to spaces of homogeneous type can be found in [11].

Theorem 2.9. [19] If $T$ is a Calderón-Zygmund singular integral operator and extends to be a bounded operator on $L^{2}\left(\mathbb{R}^{n}\right)$ and $T(1)=T^{\star}(1)=0$, then there exists a constant $C$ such that

$$
\|T f\|_{M\left(\beta^{\prime}, \gamma^{\prime}, r\right)} \leq C\|f\|_{M(\beta, \gamma, r)}
$$

for $0<\beta^{\prime}<\beta<\epsilon, 0<\gamma^{\prime}<\gamma<\epsilon$, and all $r>0$.

\section{Proof of Theorem 1.2}

Let $\phi_{j}(z, t)=2^{j(2 n+2)} \phi\left(2^{j} z, 2^{2 j} t\right)$, and $S_{j}(f)(x)=\phi_{j}{ }^{\star} f(x)$. Then $\left\{S_{j}\right\}$ is an $\epsilon$-approximation to the identity satisfying the double Lipschitz condition (2.6), and the following properties

$$
\begin{aligned}
& \lim _{j \rightarrow \infty} S_{j}=I, \quad \text { the identity operator on } L^{2}\left(\mathbb{H}^{n}\right) \\
& \lim _{j \rightarrow-\infty} S_{j}=0,
\end{aligned}
$$

both in the strong operator topology on $\mathscr{B}\left(L^{2}\left(\mathbb{H}^{n}\right)\right)$. Set $D_{j}=S_{j+1}-S_{j}$ and denote $D_{j}(f)(x)=\psi_{j}$ * $f(x)$, where $\psi_{j}(x)=2^{j(2 n+2)} \psi\left(2^{j} z, 2^{2 j} t\right)$ and $\psi(z, t)=\phi(z, t)-\phi_{1}(z, t)$. Then we will apply Coifman's decomposition of the identity operator as follows.

$$
f(z, t)=\lim _{j \rightarrow \infty} S_{j}(f)(z, t)=\sum_{j \in \mathbb{Z}} D_{j}(f)(z, t)=\sum_{j \in \mathbb{Z}} \sum_{k \in \mathbb{Z}} \psi_{k}{ }^{\star} \psi_{j}{ }^{\star} f(z, t) .
$$

For any large positive integer $N$, we write

$$
f(z, t)=\sum_{j, k:|j-k| \leq N} \psi_{k}{ }^{\star} \psi_{j}^{\star} f(z, t)+\sum_{j, k:|j-k|>N} \psi_{k}{ }^{\star} \psi_{j}^{\star} f(z, t) .
$$

To prove Theorem 1.2, we write $f$ as follows:

$$
f(z, t)=\sum_{j, k:|j-k| \leq N} \psi_{k}^{\star} \psi_{j}^{\star} f(z, t)+R_{N}^{1}(f)(z, t),
$$

where $R_{N}^{1}(f)(z, t)=\sum_{j, k:|j-k|>N} \psi_{k}{ }^{\star} \psi_{j}{ }^{\star} f(z, t)$. For any $j \in \mathbb{Z}$, denote $\psi_{j}^{N}=\sum_{k:|k| \leq N} \psi_{j+k}$. We write

$$
\begin{aligned}
\sum_{j, k:|j-k| \leq N} \psi_{k}{ }^{\star} \psi_{j} \star f(z, t) & =\sum_{j} \psi_{j}^{N}{ }^{\star} \psi_{j}^{\star} f(z, t)=\sum_{j} \int_{\mathbb{H}^{n}} \psi_{j}^{N}\left((z, t) \cdot(w, t)^{-1}\right) \psi_{j} \star f(w, s) d w d s \\
& =\sum_{j} \sum_{\substack{Q \\
\ell(Q)=2^{-j-N}}} \int_{Q}^{N} \psi_{j}^{N}\left((z, t) \cdot(w, t)^{-1}\right) \psi_{j}^{\star} f(w, s) d w d s \\
& =\sum_{j} \sum_{\substack{Q \\
\ell(Q)=2^{-j-N}}}|Q| \psi_{j}^{N}\left((z, t) \cdot\left(z_{Q}, t_{Q}\right)^{-1}\right) \psi_{j}{ }^{\star} f\left(z_{Q}, t_{Q}\right)
\end{aligned}
$$




$$
\begin{gathered}
+\sum_{j} \sum_{\substack{Q \\
\ell(Q)=2^{-j-N}}} \int_{Q}\left[\psi_{j}^{N}\left((z, t) \cdot(w, s)^{-1}\right) D_{k}(f)(w, s)\right. \\
\left.\quad-\psi_{j}^{N}\left((z, t) \cdot\left(z_{Q}, t_{Q}\right)^{-1}\right) \psi_{j}{ }^{\star} f\left(z_{Q}, t_{Q}\right)\right] d w d s \\
=\quad T_{N}(f)(z, t)+R_{N}^{2}(f)(z, t),
\end{gathered}
$$

where $Q$ denotes dyadic cubes in the sense of Lemma 2.1,

$$
\begin{aligned}
T_{N}(f)(z, t)= & \sum_{j \in \mathbb{Z}} \sum_{\substack{Q \\
\ell(Q)=2^{-j-N}}}|Q| \psi_{j}^{N}\left((z, t) \cdot\left(z_{Q}, t_{Q}\right)^{-1}\right) \psi_{j} \star f\left(z_{Q}, t_{Q}\right), \\
R_{N}^{2}(f)(z, t)= & \sum_{j \in \mathbb{Z}} \sum_{\substack{Q \\
\ell(Q)=2^{-j-N}}} \int_{Q}\left[\psi_{j}^{N}\left((z, t) \cdot(w, s)^{-1}\right) \psi_{j} \star f(w, s)\right. \\
& \left.-\psi_{j}^{N}\left((z, t) \cdot\left(z_{Q}, t_{Q}\right)^{-1}\right) \psi_{j} \star f\left(z_{Q}, t_{Q}\right)\right] d w d s,
\end{aligned}
$$

and $\left(z_{Q}, t_{Q}\right)$ stands for any fixed point in $Q$.

The key estimates are the following:

$$
\begin{aligned}
\left\|R_{N}^{1}(f)\right\|_{\mathcal{M}(\beta, \gamma, r)} & \leq C 2^{-N \delta}\|f\|_{\mathcal{M}(\beta, \gamma, r)}, \\
\left\|R_{N}^{2}(f)\right\|_{\mathcal{M}(\beta, \gamma, r)} & \leq C 2^{-N \delta}\|f\|_{\mathcal{M}(\beta, \gamma, r)}
\end{aligned}
$$

for some $\delta>0,0<\beta, \gamma<\epsilon, r>0$, and $f \in \mathcal{M}(\beta, \gamma, r)$. Assuming that these estimates hold for the moment, we continue to finish the proof of Theorem 1.2. The estimates (3.1) and (3.2) imply that $T_{N}^{-1}=\sum_{l=0}^{\infty}\left(R_{N}^{1}+R_{N}^{2}\right)^{l}$ and hence $T_{N}^{-1}$ maps the test functions in $\mathcal{N}(\beta, \gamma, r)$ to $\mathcal{N}(\beta, \gamma, r)$ for $0<\beta, \gamma<\epsilon$, and $r>0$. Moreover,

$$
\left\|T_{N}^{-1}(f)\right\|_{\mathcal{M}(\beta, \gamma, r)} \leq C\|f\|_{\mathcal{M}(\beta, \gamma, r)} .
$$

Finally, we obtain

$$
\begin{aligned}
f(z, t) & =T_{N}^{-1} T_{N}(f)(z, t) \\
& =T_{N}^{-1}\left(\sum_{j} \sum_{\substack{Q \\
\ell(Q)=2^{-j-N}}}|Q| \psi_{j}^{N}\left((\cdot, \cdot) \cdot\left(z_{Q}, t_{Q}\right)^{-1}\right) \psi_{j} \star f\left(z_{Q}, t_{Q}\right)\right)(z, t) \\
& =\sum_{j} \sum_{\substack{Q \\
\ell(Q)=2^{-j-N}}}|Q| T_{N}^{-1}\left(\psi_{j}^{N}\left((\cdot, \cdot) \cdot\left(z_{Q}, t_{Q}\right)^{-1}\right)\right)(z, t) \psi_{j}^{\star}{ }^{\star} f\left(z_{Q}, t_{Q}\right) .
\end{aligned}
$$

Note that $\psi_{j}^{N}\left((\cdot, \cdot) \cdot\left(z_{Q}, t_{Q}\right)^{-1}\right) \in \mathcal{M}\left(\epsilon, \epsilon, 2^{-j}, z_{Q}, t_{Q}\right)$ and hence for $0<\epsilon^{\prime}<\epsilon, T_{N}^{-1}\left(\psi_{j}^{N}\left((\cdot, \cdot) \cdot\left(z_{Q}, t_{Q}\right)^{-1}\right)\right)(z, t) \in$ $\mathcal{M}\left(\epsilon^{\prime}, \epsilon^{\prime}, 2^{-j}, z_{Q}, t_{Q}\right)$.

Denote $\widetilde{\psi}_{j}\left(z, t ; z_{Q}, t_{Q}\right)=T_{N}^{-1}\left(\psi_{j}^{N}\left((\cdot, \cdot) \cdot\left(z_{Q}, t_{Q}\right)^{-1}\right)\right)(z, t)$. Then properties (i), (ii), and (iii) in Theorem 1.2 are fulfilled and

$$
f(z, t)=\sum_{j \in \mathbb{Z}} \sum_{\substack{Q \\ \ell(Q)=2^{-j-N}}}|Q| \widetilde{\psi}_{j}\left(z, t ; z_{Q}, t_{Q}\right) \psi_{j}{ }^{\star} f\left(z_{Q}, t_{Q}\right),
$$

which establishes the first equality in (1.1). The second equality in (1.1) can be obtained similarly, by interchanging the order of $T_{N}^{-1}$ and $T_{N}$ in (3.3). We omit the details.

To finish the proof, it remains to prove (3.1) and (3.2).

Proof of (3.2). We write

$$
R_{N}^{2}(f)(z, t)=\sum_{j \in \mathbb{Z}} \sum_{\substack{Q \\ \ell(Q)=2^{-j-N}}} \int_{Q} \psi_{j}^{N}\left((z, t) \cdot(w, s)^{-1}\right) \psi_{j}{ }^{\star} f(w, s) d w d s
$$




$$
\begin{aligned}
& -\sum_{j \in \mathbb{Z}} \sum_{\substack{Q \\
\ell(Q)=2^{-j-N}}} \int_{Q} \psi_{j}^{N}\left((z, t) \cdot\left(z_{Q}, t_{Q}\right)^{-1}\right) \psi_{j} \star f\left(z_{Q}, t_{Q}\right) d w d s \\
=: \quad & K_{1}(f)(z, t)+K_{2}(f)(z, t),
\end{aligned}
$$

where $K_{1}$ and $K_{2}$ are linear operators with kernels

$$
K_{1}(z, t ; \tilde{z}, \tilde{t})=\sum_{j \in \mathbb{Z}} \sum_{\substack{Q \\ \ell(Q)=2^{-j-N}}} \int_{Q} \psi_{j}^{N}\left((z, t) \cdot(w, s)^{-1}\right) \psi_{j}\left((w, s) \cdot(\tilde{z}, \tilde{t})^{-1}\right) d w d s
$$

and

$$
K_{2}(z, t ; \tilde{z}, \tilde{t})=\sum_{j \in \mathbb{Z}} \sum_{\substack{Q \\ \ell(Q)=2^{-j-N}}} \int_{Q} \psi_{j}^{N}\left((z, t) \cdot\left(z_{Q}, t_{Q}\right)^{-1}\right) \psi_{j}\left(\left(z_{Q}, t_{Q}\right) \cdot(\tilde{z}, \tilde{t})^{-1}\right) d w d s .
$$

By Theorem 2.7, it suffices to show that $K_{1}$ and $K_{2}$ are Calderón-Zygmund kernels satisfying the double difference condition (2.7), with constants of at most $C 2^{-\delta N}$, and that $K_{1}$ and $K_{2}$ are bounded on $L^{2}$ with bounds of at most $C 2^{-\delta N}$ for some $\delta>0$. The latter can be shown by arguments similar to those given in [11, 17]. We omit the details here.

Now we begin to show that $K_{1}$ and $K_{2}$ are Calderón-Zygmund kernels satisfying the double difference condition (2.7), with constants of at most $C 2^{-\delta N}$. We only work with $K_{1}$, since $K_{2}$ can be handled in the same way. Notice that $\psi_{j}^{N}$ satisfies the same size, regularity, and double difference conditions as $\psi_{j}$ with the constant $C$ replaced by $C N$. It then follows from (2.1) and (2.2) that

$$
\begin{aligned}
& \left|K_{1}(z, t ; \tilde{z}, \tilde{t})\right| \\
\leq & \sum_{j \in \mathbb{Z}} \sum_{\substack{Q \\
\ell(Q)=2^{-j-N}}} \int_{Q}\left|\psi_{j}^{N}\left((z, t) \cdot(w, s)^{-1}\right) \| \psi_{j}\left((w, s) \cdot(\tilde{z}, \tilde{t})^{-1}\right)-\psi_{j}\left(\left(z_{Q}, t_{Q}\right) \cdot(\tilde{z}, \tilde{t})^{-1}\right)\right| d w d s \\
\lesssim & N \sum_{j \in \mathbb{Z}} \sum_{\substack{Q \\
\ell(Q)=2^{-j-N}}} \int_{Q} \frac{2^{-j \epsilon}}{\left(2^{-j}+d((z, t),(w, s))\right)^{Q+\epsilon}}\left(\frac{d\left((w, s),\left(z_{Q}, t_{Q}\right)\right)}{2^{-j}+d((w, s),(\tilde{z}, \tilde{t}))}\right)^{\epsilon} \\
& \times \frac{2^{-j \epsilon}}{\left(2^{-j}+d((w, s),(\tilde{z}, \tilde{t}))\right)^{Q+\epsilon}} d w d s \\
\lesssim & 2^{-N \epsilon} \sum_{j \in \mathbb{Z}} \int_{Q} \frac{2^{-j \epsilon}}{\left(2^{-j}+d((z, t),(w, s))\right)^{Q+\epsilon}} \frac{2^{-j \epsilon}}{\left(2^{-j}+d((w, s),(\tilde{z}, \tilde{t}))\right)^{Q+\epsilon}} d w d s \\
& \quad \frac{2^{-j \epsilon}}{d((z, t),(\tilde{z}, \tilde{t}))^{Q}} \quad \text { for } 0<\delta<\epsilon,
\end{aligned}
$$

establishing the size condition of $K_{1}$.

Next, let us verify the regularity conditions for $K_{1}$. We only prove the regularity condition in the first variable as $K_{1}$ is a convolution kernel. We claim that, for $d\left((z, t),\left(z^{\prime}, t^{\prime}\right)\right) \leq \frac{1}{2 a^{2}} d((z, t),(\tilde{z}, \tilde{t}))$ and any $0<\epsilon^{\prime}<$ $\epsilon$,

$$
\begin{aligned}
& \left|\sum_{\substack{Q \\
\ell(Q)=2^{-j-N}}} \int_{Q}\left[\psi_{j}^{N}\left((z, t) \cdot(w, s)^{-1}\right)-\psi_{j}^{N}\left(\left(z^{\prime}, t^{\prime}\right) \cdot(w, s)^{-1}\right)\right] \psi_{j}\left((w, s) \cdot(\tilde{z}, \tilde{t})^{-1}\right) d w d s\right| \\
& \quad \lesssim N\left(\frac{d\left((z, t),\left(z^{\prime}, t^{\prime}\right)\right)}{2^{-j}+d((z, t),(\tilde{z}, \tilde{t})}\right)^{\epsilon} \frac{2^{-j \epsilon}}{\left(2^{-j}+d((z, t),(\tilde{z}, \tilde{t}))^{2 n+2+\epsilon}\right.}
\end{aligned}
$$

and

$$
\left|\sum_{\substack{Q \\ \ell(Q)=2^{-j-N}}} \int_{Q}\left[\psi_{j}^{N}\left((z, t) \cdot(w, s)^{-1}\right)-\psi_{j}^{N}\left(\left(z^{\prime}, t^{\prime}\right) \cdot(w, s)^{-1}\right)\right] \psi_{j}\left((w, s) \cdot(\tilde{z}, \tilde{t})^{-1}\right) d w d s\right|
$$




$$
\lesssim 2^{-N \epsilon^{\prime}} \frac{2^{-j \epsilon}}{\left(2^{-j}+d((z, t),(\tilde{z}, \tilde{t}))^{2 n+2+\epsilon}\right.} .
$$

Assume that (3.4) and (3.5) hold for the moment. By interpolation it follows that for any $0<\epsilon^{\prime}<\epsilon$ and $0<\delta<\epsilon-\epsilon^{\prime}$,

$$
\begin{aligned}
& \left|K_{1}(z, t ; \tilde{z}, \tilde{t})-K_{1}\left(z^{\prime}, t^{\prime} ; \tilde{z}, \tilde{t}\right)\right| \\
\lesssim & \sum_{j} 2^{-N \delta}\left(\frac{d\left((z, t),\left(z^{\prime}, t^{\prime}\right)\right)}{2^{-j}+d((z, t),(\tilde{z}, \tilde{t})}\right)^{\epsilon^{\prime}} \frac{2^{-j \epsilon}}{\left(2^{-j}+d((z, t),(\tilde{z}, \tilde{t}))^{Q+\epsilon}\right.} \\
\lesssim & 2^{-N \delta} d\left((z, t),\left(z^{\prime}, t^{\prime}\right)\right)^{\epsilon^{\prime}} \sum_{j} \frac{2^{-j \epsilon}}{\left(2^{-j}+d((z, t),(\tilde{z}, \tilde{t}))\right)^{Q+\epsilon+\epsilon^{\prime}}} \\
\lesssim & 2^{-N \delta} \frac{d\left((z, t),\left(z^{\prime}, t^{\prime}\right)\right)^{\epsilon^{\prime}}}{d((z, t),(\tilde{z}, \tilde{t})))^{Q+\epsilon^{\prime}}},
\end{aligned}
$$

which proves the regularity condition for $K_{1}$.

Let us now prove (3.4) and (3.5). To show (3.5), using the cancellation condition of $\psi_{j}^{N}$, we have

$$
\begin{aligned}
& \left|\sum_{\substack{Q \\
\ell(Q)=2^{-j-N}}} \int_{Q}\left[\psi_{j}^{N}\left((z, t) \cdot(w, s)^{-1}\right)-\psi_{j}^{N}\left(\left(z^{\prime}, t^{\prime}\right) \cdot(w, s)^{-1}\right)\right] \psi_{j}\left((w, s) \cdot(\tilde{z}, \tilde{t})^{-1}\right) d w d s\right| \\
& \leq \quad \sum_{\substack{Q \\
\ell(Q)=2^{-j-N}}} \int_{Q}\left[\left|\psi_{j}^{N}\left((z, t) \cdot(w, s)^{-1}\right)\right|+\left|\psi_{j}^{N}\left(\left(z^{\prime}, t^{\prime}\right) \cdot(w, s)^{-1}\right)\right|\right] \\
& \times\left|\psi_{j}\left((w, s) \cdot(\tilde{z}, \tilde{t})^{-1}\right)-\psi_{j}\left(\left(z_{Q}, t_{Q}\right) \cdot(\tilde{z}, \tilde{t})^{-1}\right)\right| d w d s \\
& \lesssim N \sum_{\substack{Q \\
\ell(Q)=2^{-j-N}}} \int_{Q}\left[\frac{2^{-j \epsilon}}{\left(2^{-j}+d((z, t),(w, s))\right)^{2 n+2+\epsilon}}+\frac{2^{-j \epsilon}}{\left(2^{-j}+d\left(\left(z^{\prime}, t^{\prime}\right),(w, s)\right)\right)^{2 n+2+\epsilon}}\right] \\
& \times\left(\frac{d\left((w, s),\left(z_{Q}, t_{Q}\right)\right)}{2^{-j}+d((w, s),(\tilde{z}, \tilde{t}))}\right)^{\epsilon} \frac{2^{-j \epsilon}}{\left(2^{-j}+d((w, s),(\tilde{z}, \tilde{t}))\right)^{2 n+2+\epsilon}} d w d s \\
& \lesssim 2^{-N \epsilon^{\prime}} \int_{W_{1}}\left[\frac{2^{-j \epsilon}}{\left(2^{-j}+d((z, t),(w, s))\right)^{2 n+2+\epsilon}}+\frac{2^{-j \epsilon}}{\left(2^{-j}+d\left(\left(z^{\prime}, t^{\prime}\right),(w, s)\right)\right)^{2 n+2+\epsilon}}\right] \\
& \frac{2^{-j \epsilon}}{\left(2^{-j}+d((w, s),(\tilde{z}, \tilde{t}))\right)^{2 n+2+\epsilon}} d w d s \\
& +2^{-N \epsilon^{\prime}} \int_{W_{2}}\left[\frac{2^{-j \epsilon}}{\left(2^{-j}+d((z, t),(w, s))\right)^{2 n+2+\epsilon}}+\frac{2^{-j \epsilon}}{\left(2^{-j}+d\left(\left(z^{\prime}, t^{\prime}\right),(w, s)\right)\right)^{2 n+2+\epsilon}}\right] \\
& \frac{2^{-j \epsilon}}{\left(2^{-j}+d((w, s),(\tilde{z}, \tilde{t}))\right)^{2 n+2+\epsilon}} d w d s \\
& =I_{1}+I_{2} \text {, }
\end{aligned}
$$

where

$$
\begin{aligned}
& W_{1}=\left\{(w, s) \in \mathbb{H}^{n}: d((w, s),(\tilde{z}, \tilde{t}))>\frac{1}{3 a} d((z, t),(\tilde{z}, \tilde{t}))\right\}, \\
& W_{2}=\left\{(w, s) \in \mathbb{H}^{n}: d((w, s),(\tilde{z}, \tilde{t})) \leq \frac{1}{3 a} d((z, t),(\tilde{z}, \tilde{t}))\right\} .
\end{aligned}
$$

For $I_{1}$, we have

$$
\begin{aligned}
I_{1} \lesssim & 2^{-N \epsilon^{\prime}} \frac{2^{-j \epsilon}}{\left(2^{-j}+d((z, t),(\tilde{z}, \tilde{t}))\right)^{2 n+2+\epsilon}} \\
& \int_{W_{1}}\left[\frac{2^{-j \epsilon}}{\left(2^{-j}+d((z, t),(w, s))\right)^{2 n+2+\epsilon}}+\frac{2^{-j \epsilon}}{\left(2^{-j}+d\left(\left(z^{\prime}, t^{\prime}\right),(w, s)\right)\right)^{2 n+2+\epsilon}}\right] d w d s
\end{aligned}
$$




$$
\lesssim \quad 2^{-N \epsilon^{\prime}} \frac{2^{-j \epsilon}}{\left(2^{-j}+d((z, t),(\tilde{z}, \tilde{t}))\right)^{2 n+2+\epsilon}} .
$$

As for $I_{2}$, noticing that for $(w, s) \in W_{2}$ and $d\left((z, t),\left(z^{\prime}, t^{\prime}\right)\right) \leq \frac{1}{2 a^{2}} d((z, t),(\tilde{z}, \tilde{t}))$, we have

$$
\begin{aligned}
d((z, t),(w, s)) & \geq(1 / a) d((z, t),(\tilde{z}, \tilde{t}))-d((\tilde{z}, \tilde{t}),(w, s)) \geq(2 / 3 a) d((z, t),(\tilde{z}, \tilde{t})), \\
d\left(\left(z^{\prime}, t^{\prime}\right),(w, s)\right) & \geq(1 / a) d((z, t),(w, s))-d\left((z, t),\left(z^{\prime}, t^{\prime}\right)\right) \geq\left(1 / 6 a^{2}\right) d((z, t),(\tilde{z}, \tilde{t})) .
\end{aligned}
$$

This implies

$$
\begin{aligned}
I_{2} & \lesssim N 2^{-N \epsilon^{\prime}} \frac{2^{-j \epsilon}}{\left(2^{-j}+d((z, t),(\tilde{z}, \tilde{t}))\right)^{2 n+2+\epsilon}} \int_{W_{2}} \frac{2^{-j \epsilon}}{\left(2^{-j}+d((w, s),(\tilde{z}, \tilde{t}))\right)^{2 n+2+\epsilon}} d w d s \\
& \lesssim 2^{-N \epsilon^{\prime}} \frac{2^{-j \epsilon}}{\left(2^{-j}+d((z, t),(\tilde{z}, \tilde{t}))\right)^{2 n+2+\epsilon}},
\end{aligned}
$$

establishing (3.5).

To prove (3.4), using the cancellation condition of $\psi_{j}^{N}(x-\cdot)-\psi_{j}^{N}\left(x^{\prime}-\cdot\right)$, we write

$$
\begin{aligned}
\text { LHS of (3.4) }= & \mid \int_{\mathbb{H}^{n}}\left[\psi_{j}^{N}\left((z, t) \cdot(w, s)^{-1}\right)-\psi_{j}^{N}\left(\left(z^{\prime}, t^{\prime}\right) \cdot(w, s)^{-1}\right)\right] \\
\times & \left.\times \psi_{j}\left((w, s) \cdot(\tilde{z}, \tilde{t})^{-1}\right)-\psi_{j}\left((z, t) \cdot(\tilde{z}, \tilde{t})^{-1}\right)\right] d w d s \mid \\
= & \mid \int_{\mathbb{H}^{n}}\left[\psi_{j}^{N}\left((z, t) \cdot(w, s)^{-1}\right)-\psi_{j}^{N}\left(\left(z^{\prime}, t^{\prime}\right) \cdot(w, s)^{-1}\right)\right] \\
+ & \times \int_{\mathbb{H}^{n}}\left[\psi_{j}^{N}\left((z, t) \cdot(w, s)^{-1}\right)-\psi_{j}^{N}\left(\left(z^{\prime}, t^{\prime}\right) \cdot(w, s)^{-1}\right)\right] \\
& \times\left[\psi_{j}\left((w, s) \cdot(\tilde{z}, \tilde{t})^{-1}\right)-\psi_{j}\left((z, t) \cdot(\tilde{z}, \tilde{t})^{-1}\right)\right] d w d s \mid \\
& +\mid \int_{\mathbb{H}^{n}}\left[\psi_{j}^{N}\left((z, t) \cdot(w, s)^{-1}\right)-\psi_{j}^{N}\left(\left(z^{\prime}, t^{\prime}\right) \cdot(w, s)^{-1}\right)\right] \\
& \times\left[\psi_{j}\left((w, s) \cdot(\tilde{z}, \tilde{t})^{-1}\right)-\psi_{j}\left((z, t) \cdot(\tilde{z}, \tilde{t})^{-1}\right)\right] d w d s \mid \\
=: \quad & I I_{1}+I I_{2}+I I_{3},
\end{aligned}
$$

where

$$
\begin{aligned}
& U_{1}=\left\{z \in \mathbb{H}^{n}: d\left((z, t),\left(z^{\prime}, t^{\prime}\right)\right) \leq \frac{1}{4 a^{2}}\left(2^{-j}+d((z, t),(\tilde{z}, \tilde{t}))\right) \leq \frac{1}{2 a}\left(2^{-j}+d((z, t),(w, s))\right)\right\}, \\
& U_{2}=\left\{z \in \mathbb{H}^{n}: d\left((z, t),\left(z^{\prime}, t^{\prime}\right)\right) \leq \frac{1}{2 a}\left(2^{-j}+d((z, t),(w, s))\right) \leq \frac{1}{4 a^{2}}\left(2^{-j}+d((z, t),(\tilde{z}, \tilde{t}))\right)\right\}, \\
& U_{3}=\left\{z \in \mathbb{H}^{n}: d\left((z, t),\left(z^{\prime}, t^{\prime}\right)\right)>\frac{1}{2 a}\left(2^{-j}+d((z, t),(w, s))\right)\right\} .
\end{aligned}
$$

For $I I_{1}$, using the estimates (2.1) and (2.2), we get

$$
\begin{aligned}
I I_{1} \lesssim & N \int_{U_{1}}\left(\frac{d\left((z, t),\left(z^{\prime}, t^{\prime}\right)\right)}{2^{-j}+d((z, t),(w, s))}\right)^{\epsilon} \frac{2^{-j \epsilon}}{\left(2^{-j}+d((z, t),(w, s))\right)^{2 n+2+\epsilon}} \\
& \cdot\left[\frac{2^{-j \epsilon}}{\left(2^{-j}+d((w, s),(\tilde{z}, \tilde{t}))\right)^{2 n+2+\epsilon}}+\frac{2^{-j \epsilon}}{\left(2^{-j}+d((z, t),(\tilde{z}, \tilde{t}))\right)^{2 n+2+\epsilon}}\right] d w d s \\
\lesssim & N\left(\frac{d\left((z, t),\left(z^{\prime}, t^{\prime}\right)\right)}{2^{-j}+d((z, t),(\tilde{z}, \tilde{t}))}\right)^{\epsilon} \frac{2^{-j \epsilon}}{\left(2^{-j}+d((z, t),(\tilde{z}, \tilde{t}))\right)^{2 n+2+\epsilon}}
\end{aligned}
$$




$$
\begin{aligned}
& \cdot \int_{U_{1}} \frac{2^{-j \epsilon}}{\left(2^{-j}+d((w, s),(\tilde{z}, \tilde{t}))\right)^{2 n+2+\epsilon}} d w d s \\
&+ N\left(\frac{d\left((z, t),\left(z^{\prime}, t^{\prime}\right)\right)}{2^{-j}+d((z, t),(\tilde{z}, \tilde{t}))}\right)^{\epsilon} \frac{2^{-j \epsilon}}{\left(2^{-j}+d((z, t),(\tilde{z}, \tilde{t}))\right)^{2 n+2+\epsilon}} \\
& \cdot \int_{U_{1}} \frac{2^{-j \epsilon}}{\left(2^{-j}+d((z, t),(w, s))\right)^{2 n+2+\epsilon}} d w d s \\
& \lesssim \quad N\left(\frac{d\left((z, t),\left(z^{\prime}, t^{\prime}\right)\right)}{2^{-j}+d((z, t),(\tilde{z}, \tilde{t}))}\right)^{\epsilon} \frac{2^{-j \epsilon}}{\left(2^{-j}+d((z, t),(\tilde{z}, \tilde{t}))\right)^{2 n+2+\epsilon}} .
\end{aligned}
$$

Note that if $(w, s) \in U_{2}$, then $d((z, t),(w, s)) \leq(1 / 2 a)\left(2^{-j}+d((z, t),(\tilde{z}, \tilde{t}))\right)$. Using the estimate (2.2),

$$
\begin{aligned}
I I_{2} \lesssim N \int_{U_{2}}\left(\frac{d\left((z, t),\left(z^{\prime}, t^{\prime}\right)\right)}{2^{-j}+d((z, t),(w, s))}\right)^{\epsilon} \frac{2^{-j \epsilon}}{\left(2^{-j}+d((z, t),(w, s))\right)^{2 n+2+\epsilon}} \\
\quad \times\left(\frac{d((z, t),(w, s))}{2^{-j}+d((z, t),(\tilde{z}, \tilde{t}))}\right)^{\epsilon} \frac{2^{-j \epsilon}}{\left(2^{-j}+d((z, t),(\tilde{z}, \tilde{t}))\right)^{2 n+2+\epsilon}} d w d s \\
\lesssim N\left(\frac{d\left((z, t),\left(z^{\prime}, t^{\prime}\right)\right)}{2^{-j}+d((z, t),(\tilde{z}, \tilde{t}))}\right)^{\epsilon} \frac{2^{-j \epsilon}}{\left(2^{-j}+d((z, t),(\tilde{z}, \tilde{t}))\right)^{2 n+2+\epsilon}} .
\end{aligned}
$$

To estimate $I_{3}$, note that if $(w, s) \in U_{3}$, then $d((z, t),(w, s)) \leq 2 a d\left((z, t),\left(z^{\prime}, t^{\prime}\right)\right)$. By (2.2) and (2.1),

$$
\begin{aligned}
I I_{3} \lesssim & N \int_{U_{3}}\left[\frac{2^{-j \epsilon}}{\left(2^{-j}+d((z, t),(w, s))\right)^{2 n+2+\epsilon}}+\frac{2^{-j \epsilon}}{\left(2^{-j}+d\left(\left(z^{\prime}, t^{\prime}\right),(w, s)\right)\right)^{2 n+2+\epsilon}}\right] \\
& \times\left(\frac{d((z, t),(w, s))}{2^{-j}+d((z, t),(\tilde{z}, \tilde{t}))}\right)^{\epsilon} \frac{2^{-j \epsilon}}{\left(2^{-j}+d((z, t),(\tilde{z}, \tilde{t}))\right)^{2 n+2+\epsilon}} d w d s \\
\lesssim & N\left(\frac{d\left((z, t),\left(z^{\prime}, t^{\prime}\right)\right)}{2^{-j}+d((z, t),(\tilde{z}, \tilde{t}))}\right)^{\epsilon} \frac{2^{-j \epsilon}}{\left(2^{-j}+d((z, t),(\tilde{z}, \tilde{t}))\right)^{2 n+2+\epsilon}} .
\end{aligned}
$$

Putting together the estimates obtained above for $I I_{1}, I I_{2}$, and $I I_{3}$ yields (3.4), and hence the regularity condition of $K_{1}$.

Finally, let us verify the double difference condition for $K_{1}$. By Lemma 2.5, for any $\epsilon^{\prime}<\epsilon_{1}<\epsilon$,

$$
\begin{aligned}
& \mid \psi_{j}^{N \star} \psi_{j}\left((z, t) \cdot(\tilde{z}, \tilde{t})^{-1}\right)-\psi_{j}^{N} \star \psi_{j}\left(\left(z^{\prime}, t^{\prime}\right) \cdot(\tilde{z}, \tilde{t})^{-1}\right) \\
& \quad-\psi_{j}^{N \star} \psi_{j}\left((z, t) \cdot\left(\tilde{z}^{\prime}, \tilde{t}^{\prime}\right)^{-1}\right)+\psi_{j}^{N} \star \psi_{j}\left(\left(z^{\prime}, t^{\prime}\right) \cdot\left(\tilde{z}^{\prime}, \tilde{t}^{\prime}\right)^{-1}\right) \mid \\
& \quad \lesssim N\left(\frac{d\left((z, t),\left(z^{\prime}, t^{\prime}\right)\right)}{2^{-j}+d((z, t),(\tilde{z}, \tilde{t}))}\right)^{\epsilon_{1}}\left(\frac{d\left((\tilde{z}, \tilde{t}),\left(\tilde{z}^{\prime}, \tilde{t}^{\prime}\right)\right)}{2^{-j}+d((z, t),(\tilde{z}, \tilde{t}))}\right)^{\epsilon_{1}} \frac{2^{-j\left(\epsilon-\epsilon_{1}\right)}}{\left(2^{-j}+d((z, t),(\tilde{z}, \tilde{t}))\right)^{2 n+2+\left(\epsilon-\epsilon_{1}\right)}} .
\end{aligned}
$$

On the other hand, using the cancellation condition of $\psi_{j}$, we have for $d\left((z, t),\left(z^{\prime}, t^{\prime}\right)\right)<(1 / 4 a) d((z, t),(\tilde{z}, \tilde{t}))$ and $d\left((\tilde{z}, \tilde{t}),\left(\tilde{z}^{\prime}, \tilde{t}^{\prime}\right)\right)<(1 / 4 a) d((z, t),(\tilde{z}, \tilde{t}))$,

$$
\begin{aligned}
& \mid \psi_{j}^{N \star} \psi_{j}\left((z, t) \cdot(\tilde{z}, \tilde{t})^{-1}\right)-\psi_{j}^{N \star} \psi_{j}\left(\left(z^{\prime}, t^{\prime}\right) \cdot(\tilde{z}, \tilde{t})^{-1}\right) \\
& \quad-\psi_{j}^{N \star} \psi_{j}\left((z, t) \cdot\left(\tilde{z}^{\prime}, \tilde{t}^{\prime}\right)^{-1}\right)+\psi_{j}^{N} \star \psi_{j}\left(\left(z^{\prime}, t^{\prime}\right) \cdot\left(\tilde{z}^{\prime}, \tilde{t}^{\prime}\right)^{-1}\right) \mid \\
& \leq \int_{\mathbb{H}^{n}}\left|\psi_{j}^{N}\left((z, t) \cdot(w, s)^{-1}\right)\left[\psi_{j}\left((w, s) \cdot(\tilde{z}, \tilde{t})^{-1}\right)-\psi_{j}\left(\left(z_{Q}, t_{Q}\right) \cdot(\tilde{z}, \tilde{t})^{-1}\right)\right]\right| d w d s \\
& \quad+\int_{\mathbb{H}^{n}}\left|\psi_{j}^{N}\left(\left(z^{\prime}, t^{\prime}\right) \cdot(w, s)^{-1}\right)\left[\psi_{j}\left((w, s) \cdot(\tilde{z}, \tilde{t})^{-1}\right)-\psi_{j}\left(\left(z_{Q}, t_{Q}\right) \cdot(\tilde{z}, \tilde{t})^{-1}\right)\right]\right| d w d s \\
& \quad+\int_{\mathbb{H}^{n}}\left|\psi_{j}^{N}\left((z, t) \cdot(w, s)^{-1}\right)\left[\psi_{j}\left((w, s) \cdot\left(\tilde{z}^{\prime}, \tilde{t}^{\prime}\right)^{-1}\right)-\psi_{j}\left(\left(z_{Q}, t_{Q}\right) \cdot\left(\tilde{z}^{\prime}, \tilde{t}^{\prime}\right)^{-1}\right)\right]\right| d w d s \\
& \quad+\int_{\mathbb{H}^{n}}\left|\psi_{j}^{N}\left(\left(z^{\prime}, t^{\prime}\right) \cdot(w, s)^{-1}\right)\left[\psi_{j}\left((w, s) \cdot\left(\tilde{z}^{\prime}, \tilde{t}^{\prime}\right)^{-1}\right)-\psi_{j}\left(\left(z_{Q}, t_{Q}\right) \cdot\left(\tilde{z}^{\prime}, \tilde{t}^{\prime}\right)^{-1}\right)\right]\right| d w d s .
\end{aligned}
$$


Since the estimates for these terms are similar, we just work with the last one. Note that for $d\left((z, t),\left(z^{\prime}, t^{\prime}\right)\right)<$ $(1 / 4 a) d((z, t),(\tilde{z}, \tilde{t}))$ and $d\left((\tilde{z}, \tilde{t}),\left(\tilde{z}^{\prime}, \tilde{t}^{\prime}\right)\right)<(1 / 4 a) d((z, t),(\tilde{z}, \tilde{t}))$, by the triangle inequality,

$$
\begin{aligned}
d\left(\left(z^{\prime}, t^{\prime}\right),\left(\tilde{z}^{\prime}, \tilde{t}^{\prime}\right)\right) & \geq\left(1 / a^{2}\right) d((z, t),(\tilde{z}, \tilde{t}))-d\left((z, t),\left(z^{\prime}, t^{\prime}\right)\right)-d\left((\tilde{z}, \tilde{t}),\left(\tilde{z}^{\prime}, \tilde{t}^{\prime}\right)\right) \\
& \geq\left(1 / 2 a^{2}\right) d((z, t),(\tilde{z}, \tilde{t})) .
\end{aligned}
$$

Hence, for any $0<\epsilon^{\prime \prime}<\epsilon$,

$$
\begin{aligned}
& \int_{\mathbb{H}^{n}}\left|\psi_{j}^{N}\left(\left(z^{\prime}, t^{\prime}\right) \cdot(w, s)^{-1}\right)\left[\psi_{j}\left((w, s) \cdot\left(\tilde{z}^{\prime}, \tilde{t}^{\prime}\right)^{-1}\right)-\psi_{j}\left(\left(z_{Q}, t_{Q}\right) \cdot\left(\tilde{z}^{\prime}, \tilde{t}^{\prime}\right)^{-1}\right)\right]\right| d w d s \\
\lesssim & N \int_{\mathbb{H}^{n}} \frac{2^{-j \epsilon}}{\left(2^{-j}+d\left(\left(z^{\prime}, t^{\prime}\right),(w, s)\right)\right)^{2 n+2+\epsilon}}\left(\frac{d\left((w, s),\left(z_{Q}, t_{Q}\right)\right)}{2^{-j}+d\left((w, s),\left(\tilde{z}^{\prime}, \tilde{t}^{\prime}\right)\right)}\right)^{\epsilon} \\
& \times \frac{2^{-j \epsilon}}{\left(2^{-j}+d\left((w, s),\left(\tilde{z}^{\prime}, \tilde{t}^{\prime}\right)\right)\right)^{2 n+2+\epsilon}} d w d s \\
\lesssim & 2^{-N \epsilon^{\prime \prime}} \int_{\mathbb{H}^{n}} \frac{2^{-j \epsilon}}{\left(2^{-j}+d\left(\left(z^{\prime}, t^{\prime}\right),(w, s)\right)\right)^{2 n+2+\epsilon}} \frac{2^{-j \epsilon}}{\left(2^{-j}+d\left((w, s),\left(\tilde{z}^{\prime}, \tilde{t}^{\prime}\right)\right)\right)^{2 n+2+\epsilon}} d w d s \\
\lesssim & 2^{-N \epsilon^{\prime \prime}} \frac{2^{-j \epsilon}}{\left(2^{-j}+d\left(\left(z^{\prime}, t^{\prime}\right),\left(\tilde{z}^{\prime}, \tilde{t}^{\prime}\right)\right)\right)^{2 n+2+\epsilon}} \\
&
\end{aligned}
$$

Similarly, we can manipulate the other terms and obtain the same bound. Therefore

$$
\begin{aligned}
& \mid \psi_{j}^{N \star} \psi_{j}\left((z, t) \cdot(\tilde{z}, \tilde{t})^{-1}\right)-\psi_{j}^{N \star} \psi_{j}\left(\left(z^{\prime}, t^{\prime}\right) \cdot(\tilde{z}, \tilde{t})^{-1}\right) \\
& \quad-\psi_{j}^{N \star} \psi_{j}\left((z, t) \cdot\left(\tilde{z}^{\prime}, \tilde{t}^{\prime}\right)^{-1}\right)+\psi_{j}^{N \star} \psi_{j}\left(\left(z^{\prime}, t^{\prime}\right) \cdot\left(\tilde{z}^{\prime}, \tilde{t}^{\prime}\right)^{-1}\right) \mid \\
& \lesssim 2^{-N \epsilon^{\prime \prime}} \frac{2^{-j\left(\epsilon-\epsilon_{1}\right)}}{\left(2^{-j}+d((z, t),(\tilde{z}, \tilde{t}))\right)^{2 n+2+\left(\epsilon-\epsilon_{1}\right)}} .
\end{aligned}
$$

Let $\theta=\frac{\epsilon^{\prime}}{\epsilon_{1}} \in(0,1)$. Interpolating this with (3.6) yields

$$
\begin{aligned}
&\left|\left[K_{1}((z, t),(\tilde{z}, \tilde{t}))-K_{1}\left(\left(z^{\prime}, t^{\prime}\right),(\tilde{z}, \tilde{t})\right)\right]-\left[K_{1}\left((z, t),\left(\tilde{z}^{\prime}, \tilde{t}^{\prime}\right)\right)-K_{1}\left(\left(z^{\prime}, t^{\prime}\right),\left(\tilde{z}^{\prime}, \tilde{t}^{\prime}\right)\right)\right]\right| \\
& \lesssim \sum_{j} \mid \psi_{j}^{N \star} \psi_{j}\left((z, t) \cdot(\tilde{z}, \tilde{t})^{-1}\right)-\psi_{j}^{N \star} \psi_{j}\left(\left(z^{\prime}, t^{\prime}\right) \cdot(\tilde{z}, \tilde{t})^{-1}\right) \\
&-\psi_{j}^{N \star} \psi_{j}\left((z, t) \cdot\left(\tilde{z}^{\prime}, \tilde{t}^{\prime}\right)^{-1}\right)+\psi_{j}^{N \star} \psi_{j}\left(\left(z^{\prime}, t^{\prime}\right) \cdot\left(\tilde{z}^{\prime}, \tilde{t}^{\prime}\right)^{-1}\right) \mid \\
& \lesssim \quad 2^{-N \epsilon^{\prime \prime}(1-\theta)} \sum_{j \in \mathbb{Z}}\left(\frac{d\left((z, t),\left(z^{\prime}, t^{\prime}\right)\right)}{2^{-j}+d((z, t),(\tilde{z}, \tilde{t}))}\right)^{\theta \epsilon_{1}}\left(\frac{d\left((\tilde{z}, \tilde{t}),\left(\tilde{z}^{\prime}, \tilde{t}^{\prime}\right)\right)}{2^{-j}+d((z, t),(\tilde{z}, \tilde{t}))}\right)^{\theta \epsilon_{1}} \frac{2^{-j\left(\epsilon-\epsilon_{1}\right)}}{\left(2^{-j}+d((z, t),(\tilde{z}, \tilde{t}))\right)^{2 n+2+\epsilon-\epsilon_{1}}} \\
& \lesssim \quad 2^{-N \epsilon^{\prime \prime}(1-\theta)} \frac{d\left((z, t),\left(z^{\prime}, t^{\prime}\right)\right)^{\epsilon^{\prime}} d\left((\tilde{z}, \tilde{t}),\left(\tilde{z}^{\prime}, \tilde{t}^{\prime}\right)\right)^{\epsilon^{\prime}}}{d((z, t),(\tilde{z}, \tilde{t}))^{2 n+2+2 \epsilon^{\prime}}} .
\end{aligned}
$$

This concludes the proof of (3.2).

Proof of (3.1). We write

$$
\left|R_{N}^{1}((z, t),(\tilde{z}, \tilde{t}))\right|=\left|\sum_{|l|>N} \sum_{k} \psi_{k+l}{ }^{\star} \psi_{k}\left((z, t) \cdot(\tilde{z}, \tilde{t})^{-1}\right)\right| \leq \sum_{|l|>N} \sum_{k}\left|\psi_{k+l}{ }^{\star} \psi_{k}\left((z, t) \cdot(\tilde{z}, \tilde{t})^{-1}\right)\right| .
$$

By Lemma 2.3, we have

$$
\left|R_{N}^{1}((z, t),(\tilde{z}, \tilde{t}))\right| \lesssim \sum_{|l|>N} \sum_{k} 2^{-|l| \epsilon^{\prime}} \frac{2^{-((k+l) \wedge k) \epsilon}}{\left(2^{-((k+l) \wedge k)}+d((z, t),(\tilde{z}, \tilde{t}))\right)^{2 n+2+\epsilon}}
$$




$$
\lesssim 2^{-N \epsilon^{\prime}} d((z, t),(\tilde{z}, \tilde{t}))^{-2 n-2}
$$

which gives the size condition.

To show the regularity condition, by Lemma 2.3 , for $d\left((z, t),\left(z^{\prime}, t^{\prime}\right)\right) \leq \frac{1}{2 a} d((z, t),(\tilde{z}, \tilde{t}))$ we get

$$
\begin{aligned}
& \left|\psi_{k+l}{ }^{\star} \psi_{k}\left((z, t) \cdot(\tilde{z}, \tilde{t})^{-1}\right)-\psi_{k+l}{ }^{\star} \psi_{k}\left(\left(z^{\prime}, t^{\prime}\right) \cdot(\tilde{z}, \tilde{t})^{-1}\right)\right| \\
& \quad \leq C 2^{-|l| \delta}\left(\frac{d\left((z, t),\left(z^{\prime}, t^{\prime}\right)\right)}{2^{-((k+l) \wedge k)}+d((z, t),(\tilde{z}, \tilde{t}))}\right)^{\epsilon^{\prime}} \frac{2^{-((k+l) \wedge k) \epsilon^{\prime}}}{\left(2^{-((k+l) \wedge k)}+d((z, t),(\tilde{z}, \tilde{t}))\right)^{2 n+2+\epsilon^{\prime}}}
\end{aligned}
$$

where $0<\epsilon^{\prime}<\epsilon$ and $\delta>0$. Thus, for $d\left((z, t),\left(z^{\prime}, t^{\prime}\right)\right) \leq \frac{1}{2 a} d((z, t),(\tilde{z}, \tilde{t}))$,

$$
\begin{aligned}
& \left|R_{N}^{1}(z, t ; \tilde{z}, \tilde{t})-R_{N}^{1}\left(z^{\prime}, t^{\prime} ; \tilde{z}, \tilde{t}\right)\right| \\
\leq & \sum_{|l|>N} \sum_{k}\left|\psi_{k+l}{ }^{\star} \psi_{k}\left((z, t) \cdot(\tilde{z}, \tilde{t})^{-1}\right)-\psi_{k+l}{ }^{\star} \psi_{k}\left(\left(z^{\prime}, t^{\prime}\right) \cdot(\tilde{z}, \tilde{t})^{-1}\right)\right| \\
\leq & C \sum_{|l|>N} \sum_{k} 2^{-|l| \delta}\left(\frac{d\left((z, t),\left(z^{\prime}, t^{\prime}\right)\right)}{2^{-(k \wedge l)}+d((z, t),(\tilde{z}, \tilde{t}))}\right)^{\epsilon^{\prime}} \frac{2^{-(k \wedge l) \epsilon^{\prime}}}{\left(2^{-(k \wedge l)}+d((z, t),(\tilde{z}, \tilde{t}))\right)^{2 n+2+\epsilon^{\prime}}} \\
\leq & C 2^{-N \delta} d\left((z, t),\left(z^{\prime}, t^{\prime}\right)\right)^{\epsilon^{\prime}} d((z, t),(\tilde{z}, \tilde{t}))^{-\left(2 n+2+\epsilon^{\prime}\right)} .
\end{aligned}
$$

The proof of the smoothness condition in the second variable is the same. By Lemma 2.3, we obtain that for $d\left((\tilde{z}, \tilde{t}),\left(\tilde{z}^{\prime}, \tilde{t}^{\prime}\right)\right) \leq \frac{1}{2 a} d((z, t),(\tilde{z}, \tilde{t}))$,

$$
\begin{aligned}
& \left|\psi_{k+l}{ }^{\star} \psi_{k}\left((z, t) \cdot(w, s)^{-1}\right)-\psi_{k+l}{ }^{\star} \psi_{k}\left(\left(z^{\prime}, t^{\prime}\right) \cdot(w, s)^{-1}\right)\right| \\
& \quad \leq C 2^{-|l| \delta}\left(\frac{d\left((z, t),\left(z^{\prime}, t^{\prime}\right)\right)}{2^{-((k+l) \wedge k)}+d((z, t),(w, s))}\right)^{\epsilon^{\prime}} \frac{2^{-((k+l) \wedge k) \epsilon^{\prime}}}{\left(2^{-((k+l) \wedge k)}+d((z, t),(w, s))\right)^{2 n+2+\epsilon^{\prime}}}
\end{aligned}
$$

where $0<\epsilon^{\prime}<\epsilon$ and $\delta>0$. Thus, for $d\left((\tilde{z}, \tilde{t}),\left(\tilde{z}^{\prime}, \tilde{t}^{\prime}\right)\right) \leq \frac{1}{2 a} d((z, t),(\tilde{z}, \tilde{t}))$,

$$
\begin{aligned}
& \left|R_{N}^{1}(z, t ; \tilde{z}, \tilde{t})-R_{N}^{1}\left(z, t ; \tilde{z}^{\prime}, \tilde{t}^{\prime}\right)\right| \\
\leq & \sum_{|l|>N} \sum_{k}\left|\psi_{k+l}{ }^{\star} \psi_{k}\left((z, t) \cdot(\tilde{z}, \tilde{t})^{-1}\right)-\psi_{k+l}{ }^{\star} \psi_{k}\left((z, t) \cdot\left(\tilde{z}^{\prime}, \tilde{t}^{\prime}\right)^{-1}\right)\right| \\
\leq & C \sum_{|l|>N} \sum_{k} 2^{-|l| \delta}\left(\frac{d\left((\tilde{z}, \tilde{t}),\left(\tilde{z}^{\prime}, \tilde{t}^{\prime}\right)\right)}{2^{-(k \wedge l)}+d((z, t),(\tilde{z}, \tilde{t}))}\right)^{\epsilon^{\prime}} \frac{2^{-(k \wedge l) \epsilon^{\prime}}}{\left(2^{-(k \wedge l)}+d((z, t),(\tilde{z}, \tilde{t}))\right)^{2 n+2+\epsilon^{\prime}}} \\
\leq & C 2^{-N \delta} d\left((\tilde{z}, \tilde{t}),\left(\tilde{z}^{\prime}, \tilde{t}^{\prime}\right)\right)^{\epsilon^{\prime}} d((z, t),(\tilde{z}, \tilde{t}))^{-\left(2 n+2+\epsilon^{\prime}\right)} .
\end{aligned}
$$

To verify the double difference condition (2.7), by the geometric mean of the estimates in Lemma 2.5, (3.7) and (3.8), we get that for $d\left((z, t),\left(z^{\prime}, t^{\prime}\right)\right) \leq \frac{1}{2 a} d((z, t),(\tilde{z}, \tilde{t}))$, and $d\left((\tilde{z}, \tilde{t}),\left(\tilde{z}^{\prime}, \tilde{t}^{\prime}\right)\right) \leq \frac{1}{2 a} d((z, t),(\tilde{z}, \tilde{t}))$,

$$
\begin{aligned}
& \mid\left[\psi_{k+l}{ }^{\star} \psi_{k}\left((z, t) \cdot(\tilde{z}, \tilde{t})^{-1}\right)-\psi_{k+l}{ }^{\star} \psi_{k}\left(\left(z^{\prime}, t^{\prime}\right) \cdot(\tilde{z}, \tilde{t})^{-1}\right)\right] \\
& -\left[\psi_{k+l}{ }^{\star} \psi_{k}\left((z, t) \cdot\left(\tilde{z}^{\prime}, \tilde{t}^{\prime}\right)^{-1}\right)-\psi_{k+l}{ }^{\star} \psi_{k}\left(\left(z^{\prime}, t^{\prime}\right) \cdot\left(\tilde{z}^{\prime}, \tilde{t}^{\prime}\right)^{-1}\right)\right] \mid \\
& \leq C 2^{-|l| \delta}\left(\frac{d\left((z, t),\left(z^{\prime}, t^{\prime}\right)\right)}{2^{-((k+l) \wedge k)}+d((z, t),(\tilde{z}, \tilde{t}))}\right)^{\epsilon^{\prime}}\left(\frac{d\left((\tilde{z}, \tilde{t}),\left(\tilde{z}^{\prime}, \tilde{t}^{\prime}\right)\right)}{2^{-((k+l) \wedge k)}+d((z, t),(\tilde{z}, \tilde{t}))}\right)^{\epsilon^{\prime}} \\
& \times \frac{2^{-((k+l) \wedge k)\left(\epsilon-\epsilon^{\prime}\right)}}{\left(2^{-((k+l) \wedge k)}+d((z, t),(\tilde{z}, \tilde{t}))\right)^{2 n+2+\left(\epsilon-\epsilon^{\prime}\right)}} .
\end{aligned}
$$

Thus, for $d\left((z, t),\left(z^{\prime}, t^{\prime}\right)\right) \leq \frac{1}{2 a} d((z, t),(\tilde{z}, \tilde{t}))$, and $d\left((\tilde{z}, \tilde{t}),\left(\tilde{z}^{\prime}, \tilde{t}^{\prime}\right)\right) \leq \frac{1}{2 a} d((z, t),(\tilde{z}, \tilde{t}))$,

$$
\begin{aligned}
& \left|R_{N}^{1}(z, t ; \tilde{z}, \tilde{t})-R_{N}^{1}\left(z^{\prime}, t^{\prime} ; \tilde{z}, \tilde{t}\right)-R_{N}^{1}\left(z, t ; \tilde{z}^{\prime}, \tilde{t}^{\prime}\right)+R_{N}^{1}\left(z^{\prime}, t^{\prime} ; \tilde{z}^{\prime}, \tilde{t}^{\prime}\right)\right| \\
& \leq \sum_{|l|>N} \sum_{k} \|\left[\psi_{k+l}{ }^{\star} \psi_{k}\left((z, t) \cdot(\tilde{z}, \tilde{t})^{-1}\right)-\psi_{k+l}{ }^{\star} \psi_{k}\left(\left(z^{\prime}, t^{\prime}\right) \cdot(\tilde{z}, \tilde{t})^{-1}\right)\right] \\
& -\left[\psi_{k+l}{ }^{\star} \psi_{k}\left((z, t) \cdot\left(\tilde{z}^{\prime}, \tilde{t}^{\prime}\right)^{-1}\right)-\psi_{k+l}{ }^{\star} \psi_{k}\left(\left(z^{\prime}, t^{\prime}\right) \cdot\left(\tilde{z}^{\prime}, \tilde{t}^{\prime}\right)^{-1}\right)\right] \mid \\
& \leq C 2^{-|l| \delta}\left(\frac{d\left((z, t),\left(z^{\prime}, t^{\prime}\right)\right)}{2^{-((k+l) \wedge k)}+d((z, t),(\tilde{z}, \tilde{t}))}\right)^{\epsilon^{\prime}}\left(\frac{d\left((\tilde{z}, \tilde{t}),\left(\tilde{z}^{\prime}, \tilde{t}^{\prime}\right)\right)}{2^{-((k+l) \wedge k)}+d((z, t),(\tilde{z}, \tilde{t}))}\right)^{\epsilon^{\prime}}
\end{aligned}
$$




$$
\begin{gathered}
\times \frac{2^{-((k+l) \wedge k)\left(\epsilon-\epsilon^{\prime}\right)}}{\left(2^{-((k+l) \wedge k)}+d((z, t),(\tilde{z}, \tilde{t}))\right)^{2 n+2+\left(\epsilon-\epsilon^{\prime}\right)}} \\
\leq C 2^{-N \delta} d\left((z, t),\left(z^{\prime}, t^{\prime}\right)\right)^{\epsilon^{\prime}} d\left((\tilde{z}, \tilde{t}),\left(\tilde{z}^{\prime}, \tilde{t}^{\prime}\right)\right)^{\epsilon^{\prime}} d((z, t),(\tilde{z}, \tilde{t}))^{-\left(2 n+2+2 \epsilon^{\prime}\right)} .
\end{gathered}
$$

This completes the proof of (3.1) and hence Theorem 1.2 follows.

Acknowledgements: The first author is partially supported by an NSF grant DMS-1408839 and a McDevitt Endowment Fund at Georgetown University, and the third author supported by NNSF of China (Grant No. 12071473). The third author is the corresponding author.

The authors would like to thank the referee for valuable comments and suggestions, which improve their original manuscript.

\section{References}

[1] J.C. Bremer, R.R. Coifman, M. Maggioni, and A. Szlam, Diffusion wavelet packets, Appl. Comput. Harmon. Anal. 21 (1) (2006) 95-112.

[2] H.Q. Bui, and R.S. Laugesen, Wavelet frame bijectivity on Lebesgue and Hardy spaces, J. Fourier Anal. Appl. 19 (2013) 376 409.

[3] A. P. Calderón, Intermediate spaces and interpolation, the complex method, Studia Math. 24 (1964), 113-190.

[4] D.-C. Chang, Y. Han, and X. Wu, Construction of Frames Using Calderón-Zygmund Operator Theory, to appear.

[5] M. Christ, $A T(b)$ theorem with remarks on analytic capacity and the Cauchy integral, Colloq. Math. 60/61 (1990), 601-628.

[6] R.R. Coifman, A real variable characterization of $H^{p}$. Studia Math. 51 (1974), 269-274.

[7] R.R. Coifman, Wavelet analysis and signal processing, in: Signal Processing, Part I, in: IMA Vol. Math. Appl., vol. 22, Springer, New York, 1990, pp. 59-68.

[8] R.R. Coifman, and M. Maggioni, Diffusion wavelets, Appl. Comput. Harmon. Anal. 21 (1) (2006) 53-94.

[9] R.R. Coifman, and G. Weiss, Extensions of Hardy spaces and their use in analysis, Bull. Amer. Math. Soc. 83 (1977), $569-645$.

[10] G. David, J.-L. Journé and S. Semmes, Calderón-Zygmund operators, para-accretive functions and interpolation, Rev. Mat. Iberoamericana, 1 (1985), no. 4, 1-56.

[11] D. Deng, and Y. Han, Harmonic analysis on spaces of homogeneous type, Lecture Notes in Math., vol. 1966, Springer-Verlag, Berlin, 2009, with a preface by Yves Meyer.

[12] M. Frazier, and B. Jawerth, A discrete transform and decomposition of distribution spaces, J. Funct. Anal. 93 (1990), 34-170.

[13] D. Geller ANd A. MAYeli, Continuous wavelets and frames on stratified Lie groups, I. J. Fourier Anal. Appl., 12 (2006), 543579.

[14] Ya. Han, Yo. Han, and J. Li, Geometry and Hardy spaces on spaces of homogeneous type in the sense of Coifman and Weiss. Sci. China Math. 60 (2017), no. 11, 2199-2218.

[15] Y. Han, Calderón-type reproducing formula and the Tb theorem, Rev. Mat. Iberoamericana 10 (1994), 51-91.

[16] Y. Han, G. Lu and E. Sawyer, Flag Hardy spaces and Marcinkiewicz multipliers on the Heisenberg group, Anal. PDE 7 (2014), $1465-1534$.

[17] Y. Han, D. Müller and D. Yang, A theory of Besov and Triebel-Lizorkin spaces on metric measure spaces modeled on CarnotCarathéodory spaces. Abstr. Appl. Anal. 2008, Art. ID 893409, 250 pp.

[18] Y. Han, D. Müller, and D. Yang, Littlewood-Paley characterizations for Hardy spaces on spaces of homogeneous type, Math. Nach. 279 (2006), 1505-1537.

[19] Y. Meyer, Wavelets and operators Cambridge studies in advanced mathematics 37, Cambridge University Press 1992.

[20] E. M. Stein, Harmonic Analysis: Real Variable Methods, Orthogonality, and Oscillatory Integrals. Princeton University Press, 1993.

[21] M. H. Taibleson and G. Weiss, The molecular characterization of certain Hardy spaces, Astérisque 77 (1980), 67-149. 